\section{ANOS DE BIOÉTICA CLÍNICA NO HCPA: UM PIONEIRISMO QUE SE RENOVA}

\section{YEARS OF CLINICAL BIOETHICS AT HCPA: A PIONEERING SPIRIT THAT RENEWS ITSELF}

\author{
Bruna Pasqualini Genro', Fernanda Sales Luiz Vianna', \\ Gabriella dos Santos Dalmolin ${ }^{1}$, Marcia Mocellin Raymundo1, \\ José Roberto Goldim ${ }^{1}$
}

Em novembro de 1993 foi criado o Programa de Atenção aos Problemas de Bioética do Hospital de Clínicas de Porto Alegre (HCPA). O Ato no. 161/93 do presidente do HCPA, Prof. Carlos César de Albuquerque, publicado em 17 de novembro de 1993, implantou o Programa e nomeou os primeiros 11 membros do seu Grupo de Trabalho, que passou a atuar como um comitê de Bioética Clínica. O Prof. Carlos Fernando Francisconi foi nomeado como seu primeiro coordenador. Os objetivos do Programa eram: a) assessorar, como órgão consultivo, qualquer profissional da instituição que tenha, na sua área de atuação, dilemas morais; b) educar a comunidade de profissionais de saúde, alunos e eventualmente, pacientes e familiares, em questões bioéticas; c) propor normas e rotinas à Administração Central do HCPA, quando se fizer necessário ${ }^{1}$. Esta proposta estava plenamente de acordo com atividades semelhantes as praticadas em hospitais norte-americanos ${ }^{2}$.

Para ter efeito durador, o Programa tinha que ter sustentabilidade e reconhecimento. O primeiro ano do Programa foi dedicado a nivelar conhecimentos entre todos os 11 membros do Grupo de Trabalho. A contribuição do Prof. Joaquim Clotet foi fundamental para o sucesso destas atividades.

Nos primeiros anos foram publicados artigos e capítulos de livros versando sobre questões teóricas sobre a função dos Comitês de Bioética Hospitalar e a sua diferenciação das demais instâncias de avaliação de aspectos éticos e morais nas instituições ${ }^{3-7}$.

As questões envolvendo a preservação e o compartilhamento de informações referentes a pacientes levaram o Comitê de Bioética Clínica a oferecer, neste período inicial, uma atividade especificamente voltada para temas de Privacidade e Confidencialidade. Mais de 2000 colaboradores das áreas assistencial e administrativa foram capacitados ${ }^{8}$.

As consultorias de Bioética Clínica tiveram início em 1994. Elas foram demandadas por profissionais de saúde, pacientes, familiares e pela própria Administração do HCPA. Em 1997, o Serviço de Reumatologia e a Unidade de Terapia Intensiva Pediátrica (UTIP) solicitaram a realização regular de Rounds de Bioética, como forma de antecipar problemas éticos. A UTIP mantém esta atividade semanal de discussão de casos até os dias de hoje.

$\mathrm{O}$ crescimento da demanda gerou a necessidade de criar uma nova estrutura no HCPA. Em 10 de fevereiro de 2009, foi criado o Serviço de Bioética, vinculado ao Grupo de Pesquisa e Pós-Graduação (GPPG). O Serviço de Bioética passou a ser o responsável pelas consultorias de Bioética Clínica ${ }^{9}$.

Posteriormente, em 10 de março de 2015, o Grupo de Trabalho do Programa de Atenção aos Problemas de Bioética teve a sua denominação alterada para Comitê de Bioética Clínica do HCPA, por meio do Ato no. 036/2015, da Diretora Presidente do HCPA, Prof. Nadine Clausell.

O Comitê de Bioética Clínica do HCPA realizou, ao longo do período 1994-2018, 3276 consultorias. Vale destacar que 2397 foram realizadas nos últimos cinco anos, com uma média de 10,11 consultorias por semana. O tempo médio de resposta às consultorias solicitadas foi de $6 \mathrm{~h} 25 \mathrm{~min}$. Vale
Clin Biomed Res. 2018;38(3):203-205

1 Serviço de Bioética, Hospital de Clínicas de Porto Alegre (HCPA). Porto Alegre, RS, Brasil.

Autor correspondente: José Roberto Goldim jgoldim@hcpa.edu.br Hospital de Clínicas de Porto Alegre Rua Ramiro Barcelos, 2350. 90035-903, Porto Alegre, RS, Brasil. 
destacar que, desde 2009, todas as consultorias são solicitadas e respondidas diretamente no prontuário eletrônico de cada paciente, utilizando o sistema AGHUse. As consultorias foram solicitadas por 53 diferentes serviços assistenciais, com destaque para os Serviços de Pediatria, Neonatologia, Medicina Interna e Psiquiatria. A maioria destas consultorias $(71 \%)$ foi solicitada por médicos ${ }^{10}$. Os principais temas abordados envolveram a privacidade dos pacientes; a revelação de diagnóstico de doenças transmissíveis para outras pessoas; especialmente parceiros sexuais estáveis; doenças genéticas de aparecimento tardio; decisões de final de vida; reprodução assistida; cirurgias de redesignação sexual e transfusões de sangue em pacientes Testemunhas de Jeová, além de questões de relacionamentos entre profissionais ${ }^{8,11}$. Em cerca de $38 \%$ das consultorias, a principal dificuldade foi a relacionada às famílias ${ }^{12}$.

Logo após a criação do Comitê do HCPA, em 1993, outras instituições brasileiras também criaram e implantaram estruturas semelhantes, tais como o Hospital das Clínicas da Universidade de São Paulo (USP), em 1996; a Pontifícia Universidade Católica de Porto Alegre (PUCRS), em 1997; e o Instituto Nacional do Câncer (INCA), em 199913,14.

A adequação e a utilidade dos Comitês de Bioética Clínica foram reconhecidas em 2015, quando o Conselho Federal de Medicina (CFM) recomendou "a criação, o funcionamento e a participação dos médicos nos Comitês de Bioética" em todos os hospitais brasileiros ${ }^{15}$. $\mathrm{Na}$ exposição de motivos desta Recomendação, o Comitê de Bioética Clínica do HCPA foi reconhecido como pioneiro no Brasil ${ }^{15}$.

O Comitê do HCPA apoiou várias instituições na constituição de seus próprios Comitês de Bioética Clínica, tais como o Grupo Hospitalar Conceição (GHC) de Porto Alegre, o Hospital da Universidade Federal de Pelotas, o Hospital da Aeronáutica de Canoas (HACO), o Hospital Universitário de Canoas, o Hospital Materno-Infantil Presidente Vargas (HMIPV) e o Hospital de Pronto Socorro (HPS) de Porto Alegre. O caso específico do HACO mereceu uma publicação específica, pois foi o primeiro Comitê de Bioética Clínica a funcionar em um hospital militar brasileiro ${ }^{16}$.
Ao longo destes 25 anos, o Comitê de Bioética Clínica também sugeriu uma série de medidas à Administração do HCPA no sentido de enfrentar desafios, tais como: a garantia e ampliação de medidas de proteção à privacidade dos pacientes; melhorias no prontuário médico; não-discriminação em ações e processos assistenciais; direitos e deveres de acompanhantes e visitantes; agressões a profissionais de saúde; utilização de redes sociais; cuidados paliativos; relações interpessoais dos profissionais de saúde; e o atendimento de recém-nascidos de alto risco ${ }^{8,14}$.

O apoio institucional foi fundamental para o reconhecimento da atividade do Comitê de Bioética Clínica. A vinculação do Comitê à Direção Médica do HCPA abriu a possibilidade de um canal direto de comunicação e o reconhecimento desta atividade como sendo inserida junto às demais instâncias assistenciais.

Foi decisiva para o sucesso das atividades a qualidade e a dedicação dos integrantes do Comitê nestes 25 anos. Mais de 60 profissionais, vinculados ao HCPA, integraram o colegiado, como médicos, de nove diferentes especialidades, enfermeiros, farmacêuticos, fisioterapeutas, geneticistas, biólogos, nutricionistas, psicólogos, assistentes sociais, advogados e administradores. Outro destaque foi a participação dos membros externos, que foram convidados em função da necessidade de ampliar as perspectivas de reflexão. O Comitê contou com a participação de desembargadores do Tribunal de Justiça, juízes de Direito, advogados, médicos, filósofos, jornalistas, sociólogos e teólogos, que em muito contribuíram para a adequada reflexão dos problemas bioéticos discutidos.

Esta foi a trajetória do Comitê de Bioética Clínica do HCPA. Muitos novos desafios são apresentados, em função de novas tecnologias, novas relações, novos parceiros, novas percepções sobre a saúde e as suas relações com as pessoas e com a sociedade. Contudo, o principal desafio foi e será manter a qualidade das reflexões e a capacidade de responder às demandas trazidas por pacientes, familiares, profissionais de saúde, gestores e pela própria sociedade, especialmente quando da tomada de decisões que envolvem a vida e viver.

\section{Referências}

1. Francisconi CF. Programa de atenção aos problemas de bioética. Fatos em Foco. 1993;17(3):10.

2. Rosner F. Hospital medical ethics committees: a review of their development. JAMA 1985;253(18):2693-7. http://dx.doi. org/10.1001/jama.1985.03350420105027. PMid:3989945.
3. Goldim JR, Francisconi CF. Os comitês de ética hospitalar. Rev Med ATM. 1995;15(1):327-34.

4. Francisconi CF, Goldim JR, Lopes MHI. O papel dos Comitês de Bioética na humanização da assistência à saúde. Rev Bioet. 2002;10:147-57.
5. Goldim JR, Francisconi CFM. Ethics committees. In: Stanciu C, Ladas S, editors. Medical ethics. Atenas: BETA; 2002. p. 21-6.

6. Batista CC, Goldim JR, Fritscher CC. Bioética clínica: ciência e humanidade. Sci Medica. 2005;15(1):52-9. 
7. Francisconi CF, Goldim JR. Bioética clínica. In: Clotet J, Feijó AGS, Oliveira MG, editores. Bioética, uma visão panorâmica. Porto Alegre: EDIPUCRS; 2005. p. 59-83.

8. Goldim JR, Francisconi CF, Raymundo MM, Salgueiro JB. Bioethics committees: a brazilian hospital experience. In: European Association of Centres of Medical Ethics (EACME). 21th Annual Conference Book of Abstracts; 2007; Zurich; The Netherlands: EACME; 2007. p. 34.

9. Hospital de Clínicas de Porto Alegre - HCPA. Regulamento Interno do Grupo de Pesquisa e PósGraduação do Hospital de Clínicas de Porto Alegre. Porto Alegre: HCPA; 2016.
10. Melnik CS, Goldim JR. Perfil das consultorias de bioética clínica envolvendo famílias que dificultaram a resolução de problemas. Rev Bioet. 2013;21(1):113-8. http:// dx.doi.org/10.1590/S198380422013000100013.

11. Genro BP. Consultorias de bioética clínica: da teoria à prática [tese]. Porto Alegre: UFRGS; 2013.

12. Melnik CS. Relações familiares e consultorias de bioética clínica [dissertação]. Porto Alegre: UFRGS; 2012.

13. Marinho S, Costa A, Palácios M, Rego S. Implementação de comitês de bioética em hospitais universitários brasileiros: dificuldades e viabilidades. $R e v$
Bioet. 2014;22(1):105-15. http:// dx.doi.org/10.1590/S198380422014000100012 .

14. Goldim JR, Raymundo MM, Fernandes MS, Helena M, Lopes I, Kippers DJ, et al. Clinical Bioethics Committees: a Brazilian experience. Int J Bioeth. 2008;19(1-2):18192, 207. http://dx.doi.org/10.3917/ jib.191.0181. PMid:18664010.

15. Conselho Federal de Medicina (CFM). Recomendação CFM 8/2015. Recomenda a criação, o funcionamento e a participação dos médicos nos Comitês de Bioética. Brasília: CFM; 2015.

16. Goldim JR. Comitês de Bioética Clínica e Hospitais Militares. Rev Científica do Hosp Aeronáutica Canoas. 2015;1(1):53-5. 